\title{
STAR family RNA-binding protein ASD-2 regulates developmental switching of mutually exclusive alternative splicing in vivo
}

\author{
Genta Ohno, ${ }^{1}$ Masatoshi Hagiwara, ${ }^{1,2,4}$ and Hidehito Kuroyanagi ${ }^{1,2,3}$ \\ ${ }^{1}$ Laboratory of Gene Expression, School of Biomedical Science, Tokyo Medical and Dental University, Tokyo \\ 113-8510, Japan; ${ }^{2}$ Department of Functional Genomics, Medical Research Institute, Tokyo Medical and Dental University, \\ Tokyo 113-8510, Japan
}

\begin{abstract}
Alternative splicing of pre-mRNAs greatly contributes to the spatiotemporal diversity of gene expression in metazoans. However, the molecular basis of developmental regulation and the precise sequence of alternative pre-mRNA processing in vivo are poorly understood. In the present study, we focus on the developmental switching of the mutually exclusive alternative splicing of the let-2 gene of Caenorhabditis elegans from the exon 9 form in embryos to the exon 10 form in adults. By visualizing the usage of the let-2 mutually exclusive exons through differential expression of green fluorescent protein (GFP) and red fluorescent protein (RFP), we isolated several switching-defective mutants and identified the alternative splicing defective-2 (asd-2) gene, encoding a novel member of the evolutionarily conserved STAR (signal transduction activators of RNA) family of RNA-binding proteins. Comparison of the amounts of partially spliced let-2 RNAs in synchronized wild-type and asd-2 mutant worms suggested that either of the introns downstream from the let-2 mutually exclusive exons is removed prior to the removal of the upstream ones, and that asd-2 promotes biased excision of intron 10 in the late larval stages. We propose that the developmental switching between alternative sequences of intron removal determines the ratio between the mature let-2 mRNA isoforms.
\end{abstract}

[Keywords: Alternative splicing; C. elegans; reporter; development; GSG/STAR; processing intermediate]

Supplemental material is available at http://www.genesdev.org.

Received September 27, 2007; revised version accepted December 4, 2007.

Alternative pre-mRNA splicing is a crucial step of gene expression and provides proteome diversity from a limited number of genes in metazoan. Recent global studies have demonstrated that as many as two thirds of human genes have multiple alternative isoforms of mature mRNAs (Modrek and Lee 2002; Johnson et al. 2003; Blencowe 2006), and that deregulation of alternative splicing may cause hereditary diseases and cancer (Faustino and Cooper 2003; Venables 2004; Licatalosi and Darnell 2006). Many alternative splicing events are controlled in tissue- and cell-type-dependent manners (Black 2003; Matlin et al. 2005), and a variety of auxiliary transacting factors and cis-acting elements involved in the regulation of alternative splicing have been identified through biochemical, genetic, and bioinformatic analyses.

Mutually exclusive alternative splicing is a strictly

Corresponding authors.

${ }^{3}$ E-MAIL kuroyana.end@tmd.ac.jp; FAX 81-3-5803-5853.

${ }^{4}$ E-MAIL m.hagiwara.end@mri.tmd.ac.jp; FAX 81-3-5803-5836.

Article published online ahead of print. Article and publication date are

online at http://www.genesdev.org/cgi/doi/10.1101/gad.1620608. regulated form of alternative splicing; only one out of two or more candidate exons are used in the mature mRNA isoform (Smith 2005). This type of alternative splicing raises two main questions; what ensures selection of only one out of multiple exons at one time, and how the selection of the exons is switched. The most extreme example of the mutually exclusive alternative splicing is in the Dscam gene of Drosophila. It has multiple clusters of variable exons and potentially produces $>38,000$ different mRNA and protein isoforms (Schmucker et al. 2000). Graveley raised a model that interaction between the only one docking site and one of multiple selector sequences that reside in the introns upstream of the variable exons allows only one variable exon out of 48 alternative exons in the exon 6 cluster to be selected (Graveley 2005). It has been demonstrated that mutually exclusive selection of two exons of the $\alpha$-tropomyosin and the $\alpha$-actinin genes in vertebrates is enforced by the absolute incompatibility of the two adjacent exons due to the close proximity of the branchpoint(s) for the downstream exon to the donor site of the upstream exon (Smith and Nadal-Ginard 1989; Southby 
et al. 1999). The mutually exclusive selection of duplicated exons in the human INK1 gene is considered to be ensured by the incompatibility between the U2-type major intron and the U12-type minor intron (Letunic et al. 2002). Mutually exclusive exons may not necessarily be strictly regulated, but nonsense-mediated mRNA decay (NMD) may contribute to the apparent fidelity of the mutually exclusive selection when neither of the exons is a multiple of three nucleotides (Jones et al. 2001b).

Mutually exclusive alternative splicing is often regulated in a tissue-specific manner. Several trans-acting factors and multiple cis-elements have been demonstrated to regulate the mutually exclusive exons of the FGFR2, $\alpha$-actinin, and $\alpha$ - and $\beta$-tropomyosin genes in the cultured cells (Chen et al. 1999; Jones et al. 2001a; Muh et al. 2002; Baraniak et al. 2003, 2006; Gromak et al. 2003a,b; Expert-Bezancon et al. 2004; Lin and Tarn 2005; Wagner et al. 2005; Crawford and Patton 2006). However, the mechanisms of the tissue-specific mutually exclusive alternative splicing are to be evaluated in vivo.

To pave the way to identify regulators of alternative splicing in living organisms, we recently developed a transgenic reporter worm system that visualizes the tissue-specific expression profiles of each of the mutually exclusive exons through differential expression of green fluorescent protein (GFP) and red fluorescent protein (RFP) (Kuroyanagi et al. 2006). With this system, we demonstrated that the evolutionarily conserved Fox-1 subfamily of RNA-binding proteins ASD-1 (for Alterna- tive Splicing Defective-1) and FOX-1 regulate tissue-specific alternative splicing of the fibroblast growth factor (FGF) receptor gene in Caenorhabditis elegans (Kuroyanagi et al. 2006). This work provided an example of tissue-specific regulation of mutually exclusive exons in vivo, and provided evidence that the Fox-1-mediated regulation of alternative splicing (Jin et al. 2003) is conserved between vertebrates and nematodes.

The let-2 gene, encoding $\alpha 2$ (IV) collagen of C. elegans, has a unique property in that selection of its mutually exclusive exon 9 and exon 10 in body wall muscles undergoes dramatic switching along with the larval development (see Fig. 1A); in embryos, an mRNA isoform with exon 9 is exclusively expressed, while in late larval and adult stages, an mRNA isoform with exon 10 predominates (Sibley et al. 1993; Graham et al. 1997). This developmental regulation of let-2 alternative splicing is evolutionarily conserved in two distantly separated nematodes, C. elegans and Ascaris (Sibley et al. 1993; Pettitt and Kingston 1994), and it is speculated that switching of exon 9 and exon 10 alters the characteristics of basement membranes during nematode development. In the present study, we applied the transgenic alternative splicing reporter system to analyze the developmentally regulated switching mechanism of the mutually exclusive exons of the let-2 gene, and identified a novel member of the highly conserved STAR (signal transduction activators of RNA) family RNA-binding proteins, ASD-2 (for Alternative Splicing Defective-2), as a regulator of the let-2 alternative splicing.

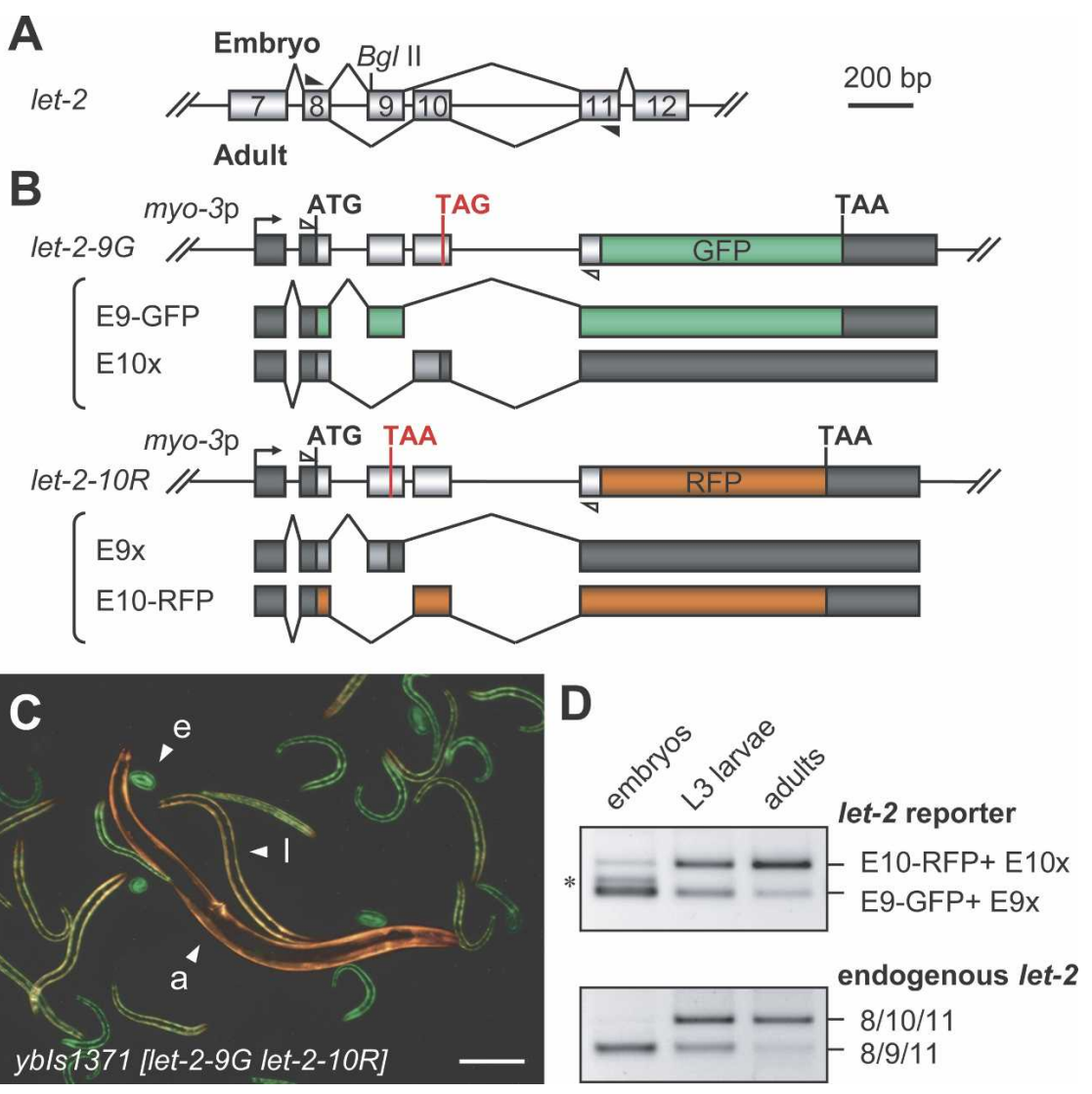

Figure 1. Visualization of the developmentally regulated mutually exclusive alternative splicing patterns of the let-2 gene. $(A)$ Schematic view of the alternatively spliced regions of the endogenous let-2 gene. Boxes indicate exons. Closed triangles indicate positions and directions of the PCR primers used to amplify the cDNA fragments from the endogenous let-2 mRNAs in $D$ and Figure 2B. $(B)$ Structure of a pair of the let-2 reporter minigenes, let-2-9G and let-2-10R, and mRNAs derived from the minigenes. Predicted ORFs are colored in green (E9GFP), red (E10-GFP), or gray (E10x and E9x). Open triangles indicate positions and directions of the PCR primers used to amplify the cDNAs derived from the let-2 reporter mRNAs in $D$ and Figure 2B. $(C)$ A microphotograph of worms with a transgenic allele ybIs1371 [myo-3::1et-2-9G myo-3::let2-10R], expressing the let-2 reporters under the myo-3 promoter. Arrowheads indicate an embryo (e), an L3 larva (1), and a young adult (a). Bar, $100 \mu \mathrm{m}$. (D) RT-PCR analysis of the let-2 reporter (top) and the endogenous let-2 (bottom). Note that BglII digested the PCR products containing exon 9 into halves. An asterisk denotes a nonspecific band. 
Ohno et al.

\section{Results}

Transgenic reporter system visualizes the developmentally regulated mutually exclusive alternative splicing of the let-2 gene in vivo

In order to monitor the selection of the let-2 mutually exclusive exons (Fig. 1A) in vivo, we constructed a pair of reporter minigenes, let-2-9G and let-2-10R (Fig. 1B). The minigenes carry the same let-2 genomic DNA fragment spanning from exon 8 to exon 11 connected in-frame to cDNAs for fluorescent proteins, and termination codons are artificially introduced into exon 10 of let-2-9G and exon 9 of let-2-10R (Fig. 1B). We expected that, from the let-2-9G minigene, selection of exon 9 would lead to expression of an mRNA encoding a GFP fusion protein (E9-GFP), while selection of exon 10 would result in a nonproductive mRNA $(\mathrm{E} 10 \mathrm{x})$ due to the termination codon (Fig. 1B). In the same way, selection of exon 10 would lead to expression of an mRNA encoding an RFP fusion protein (E10-RFP) and selection of exon 9 would result in a nonproductive mRNA $(\mathrm{E} 9 \mathrm{x})$ from the let-210R minigene (Fig. 1B).

The let-2 reporter successfully visualized the alternative exon usage. We drove expression of the let-2 reporter minigenes under the body wall muscle-specific myo-3 promoter, since the endogenous let-2 is primarily expressed in the body wall muscles (Graham et al. 1997). As expected, expression of the let-2 reporter in the body wall muscles gradually and almost completely switched from GFP to RFP along with the development; embryos exclusively express E9-GFP and elder worms express E10-RFP (Fig. 1C). RT-PCR analyses of mRNA isoforms derived from the minigenes confirmed that the alternative exons are selected mutually exclusively to produce the E9-GFP and E10x mRNA isoforms from the let-2-9G minigene, and the E9x and E10-RFP isoforms from the let-2-10R minigene (data not shown). Consistent with the color switch of the let-2 reporter worms, the composition of the reporter mRNAs gradually switched from the exon 9 forms to the exon 10 forms in parallel with that of the endogenous let-2 mRNAs along with the developmental stage (Fig. 1D). These results indicate that the let-2 reporter visualizes the developmental switching of the let-2 mutually exclusive exons in vivo.

asd-2 mutants are defective in the developmental switching of alternative splicing of both the let-2 reporter and the endogenous let-2 gene

To identify factors regulating the let-2 alternative splicing in vivo, we screened for mutants defective in the developmental switching of the let-2 alternative splicing reporter expression in the body wall muscles. We mutagenized the transgenic reporter allele ybIs1371 (Fig. 1C) and screened $\sim 5 \times 10^{4}$ genomes for F2 progeny that remain green until adulthood or that turn red as younger larvae. We isolated several independent mutant alleles, all of which remain green during larval development but eventually turn red in the senile stage (Fig. 2A; data not shown). RT-PCR analysis of the let-2 reporter confirmed that the mutants are defective in the switching to the exon 10 forms in the late larval stage (Fig. 2B, top panel) in parallel with the defect in the color switch. Furthermore, the mutants are also defective in the exon switching of the endogenous let-2 mRNAs (Fig. 2B, bottom panel), indicating that the let-2 reporter reflected the expression of the endogenous let-2 gene and allowed the isolation of alternative splicing defective mutants.

Single nucleotide polymorphism (SNP)-based chromosome mapping and genomic DNA sequencing suggested that the mutants are alleles of a single uncharacterized gene, T21G5.5 (see below). RNAi of the gene reproduced the color phenotype (Fig. 2C), confirming that the defect in the switching of the let-2 reporter expression resulted from loss or reduction of function of the gene. We therefore named it alternative splicing defective-2 (asd-2). Figure $2 \mathrm{D}$ represents a schematic structure of the asd-2 gene and summarizes the mutations identified in the asd-2 mutant alleles. Expressed sequence tag (EST) analysis has predicted that the asd-2 gene produces two protein isoforms, ASD-2a and ASD-2b, that differ in the $\mathrm{N}$ terminus sequence due to alternative use of the first exons (Fig. 2D), and we confirmed expression of both of the isoforms by cloning RT-PCR products (deposited to GenBank under accession nos. EF630625 and EF630626).

\section{ASD-2 is a novel member of the evolutionarily conserved STAR family}

ASD-2 belongs to the evolutionarily conserved STAR family of RNA-binding proteins (Vernet and Artzt 1997). The family members share a highly conserved tripartite GSG (for GRP33, Sam68, GLD-1)/STAR domain (Jones and Schedl 1995; Vernet and Artzt 1997) composed of a single KH-type RNA-binding domain and two flanking domains called QUA1 and QUA2 (Fig. 3A). QUA1 is required and sufficient for homodimer formation of C. elegans GLD-1 and mouse QKI (Chen and Richard 1998; Ryder et al. 2004). QUA2 is involved in the RNA binding together with the KH domain for GLD-1 and human SF1 (Liu et al. 2001; Ryder et al. 2004; Lehmann-Blount and Williamson 2005). Figure 3B shows the phylogenetic tree of the GSG/STAR domains of the STAR family proteins from C. elegans and mammals and Drosophila Held Out Wings (HOW). ASD-2 GSG/STAR domain has 68\%, $66 \%$, and $74 \%$ identity with those of GLD-1, QKI, and HOW, respectively, while the ASD-2 KH-QUA2 domains have only $37 \%$ identity to those of human SF1. These sequence analyses indicated that ASD-2 belongs to the GLD-1 subfamily that includes QKI and HOW.

Mutations identified in the asd-2 mutants affect conserved residues in the GSG/STAR domain or the entire structure of the ASD-2b protein. Five missense mutations were assigned to the conserved residues within the GSG/STAR domain (Fig. 3A). yb1422 causes G125E substitution within the QUA1 domain. yb1415, yb1470, and yb1419 cause R154C, H193Y, and L202F substitutions within the KH RNA-binding domain, respectively. yb1423 causes E244K substitution within the QUA2 do- 

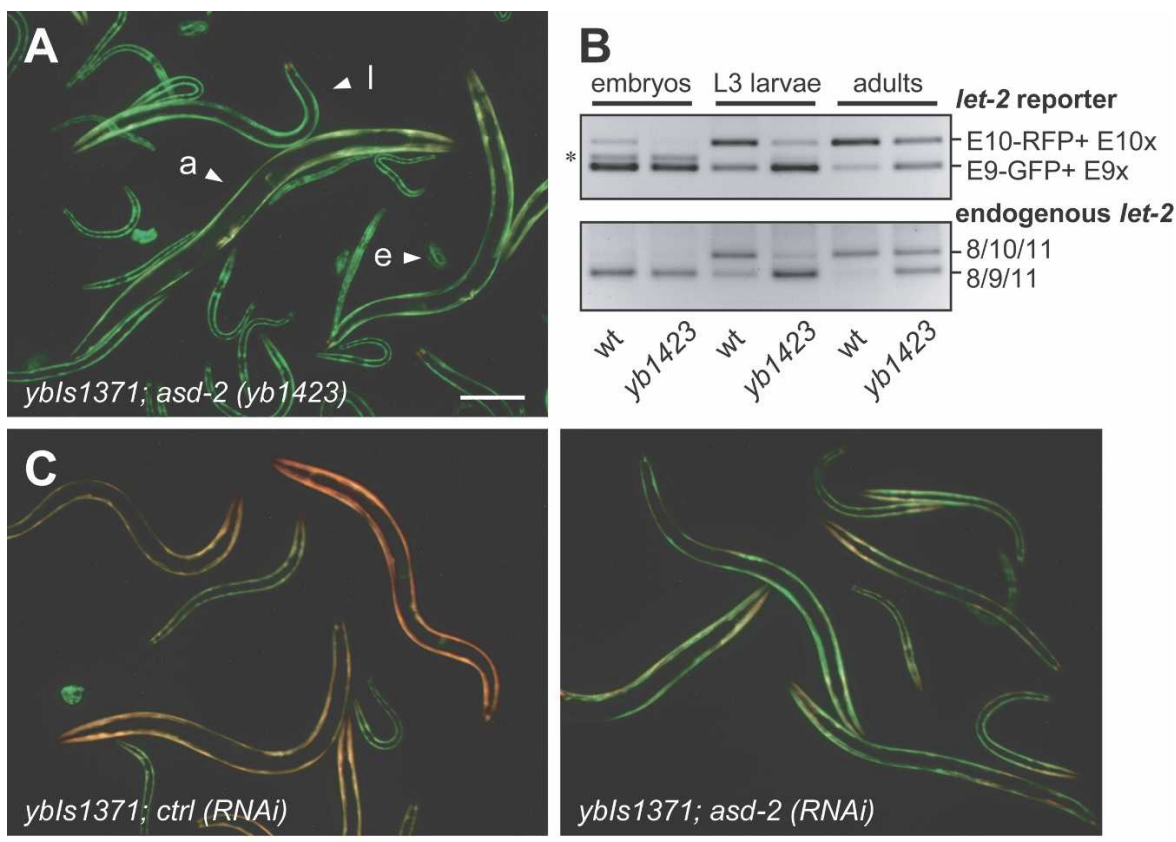

D

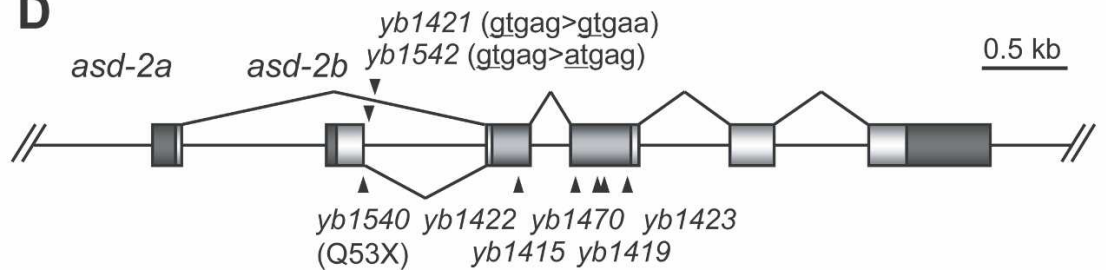

Figure 2. The asd-2 mutants defective in the developmental switching of the let-2 alternative splicing. (A) A microphotograph of a mutant allele, ybIs1371; asd-2 (yb1423). Arrowheads indicate an embryo (e), an L3 larva (1), and a young adult (a). (B) RT-PCR analysis of the let-2 reporter (top) and the endogenous let-2 (bottom). The genotypic backgrounds and the stages of the worms are indicated. An asterisk denotes a nonspecific band. (wt) Wild type. (C) RNAi of the asd-2 gene. Microphotographs of the F1 progeny of the ybIs1371 reporter worms treated with buffer alone (left panel) or with asd-2 dsRNA (right panel). Bar in $A, 100 \mu m$. (D) Schematic representation of the asd-2 gene. Boxes indicate exons. The ORFs are in white. The GSG/STAR domain is in light gray. UTRs are in dark gray. Mutations identified in the mutant alleles are indicated.

main. Putative $a s d$-2b-specific null mutations were identified in the other three alleles. yb1540 causes nonsense mutation in the first exon of the $a s d-2 b$ isoform (Fig. 2D). yb1421 and yb1542 have base substitutions at the splice donor site in the first intron of the $a s d-2 b$ isoform (Fig. 2D). These results suggested that the RNAbinding property of the ASD-2b isoform is responsible for the developmental switching of the let-2 mutually exclusive exons in the body wall muscles.

\section{ASD-2b regulates the switching of the let-2 reporter in the body wall muscles}

We then analyzed the expression profiles of the asd-2a and $a s d-2 b$ isoforms. We constructed transcriptional fusion minigenes, asd-2a::GFP and asd-2b::RFP (Fig. 4A). These reporters showed differential tissue-specific expression profiles throughout development (Fig. 4B). Expression of asd-2a::GFP was detected in hypodermis and pharyngeal muscles. On the other hand, expression of asd-2b::RFP was observed in the body wall muscles and the pharyngeal muscles, consistent with the idea that $a s d-2 b$ is responsible for the regulation of the let-2 alternative splicing in the body wall muscles.

Next, we asked whether expression of ASD-2b in the body wall muscle rescues the color phenotype of the asd-2 mutant. Overexpression of ASD-2b under the body wall muscle-specific myo-3 promoter overrescued the asd-2 (yb1423) mutant, and enforced E10-RFP expression even in early developmental stages (Fig. 4C). Figure 4D summarizes the effects of the asd-2 expression on the let-2 reporter. We concluded that the ASD-2b isoform regulates the let-2 alternative splicing reporter in the body wall muscles.

ASD-2b regulates the switching of the let-2 alternative exons via a short element in intron 10

Then, we searched for a cis-acting element for the ASD$2 \mathrm{~b}$ function in the let-2 pre-mRNA processing. Kabat et al. (2006) have recently reported that evolutionarily conserved intronic stretches are likely to be involved in the 
Ohno et al.

Figure 3. GSG/STAR domain of ASD-2 and other STAR family proteins. $(A)$ Amino acid sequence alignment of the GSG/STAR domains. Identical amino acid residues are shaded in black. Amino acid residues with similar properties are shaded in gray. The GSG/STAR domain and the QUA1 and QUA2 domains are indicated with black and gray lines, respectively, above the sequence alignment. Closed triangles indicate the positions of the missense mutations identified in asd-2 mutant alleles. An open triangle indicates the position of the E48 residue of mouse QKI essential for dimerization (Chen and Richard 1998). Asterisks under the sequences indicate the human SF1 amino acid residues contacting the branchpoint RNA 5'AUACUAACAA-3' (Liu et al. 2001). (Ce GLD-1) C. elegans GLD-1 (Francis et al. 1995); (Dm HOW) Drosophila HOW (Zaffran et al. 1997); (Ms QKI) mouse QKI (Ebersole et al. 1996); (Hs Sam68) human Sam68 (Matter et al. 2002); (Hs SF1) human SF1 (Kramer and Utans 1991). (B) Phylogenetic tree of the GSG/STAR domains from the STAR family proteins. (Ce) C. elegans; (Dm) Drosophila; (Ms) mouse; (Hs) human.
A
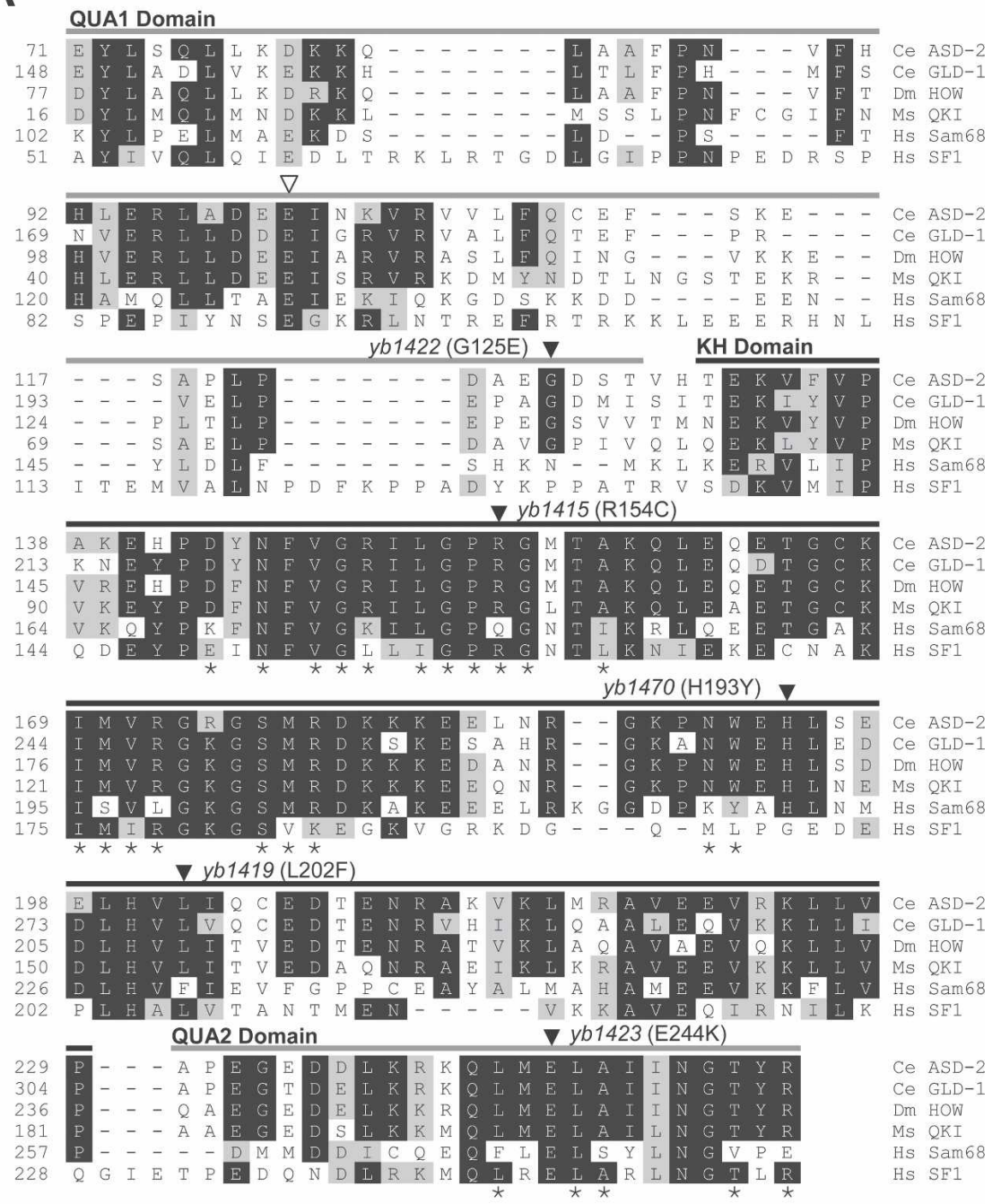

B

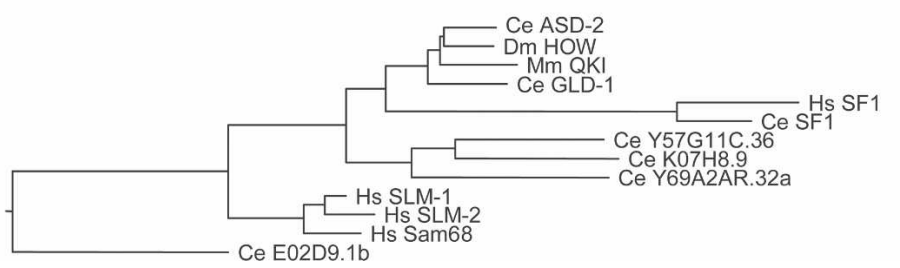

regulation of alternative pre-mRNA splicing in nematodes. Furthermore, they demonstrated that deletion of short stretches within let-2 intron 10 affected the alternative splicing regulation (Kabat et al. 2006). We therefore constructed a pair of modified let-2 reporter minigenes (M1) with a deletion of a short sequence in intron 10 (Fig. 5A), and observed that the M1 mutant reporter continued expressing E9-GFP in the late larval stages (Fig. 5B). Since the sequence deleted in the M1 minigenes contained a half-site of the QKI response element (QRE, NACUAAY-N ${ }_{1-20}-\mathrm{UAAY}$ ), a consensus sequence of mouse QKI (Galarneau and Richard 2005), we modified two residues in the short stretch, CUAACUCUAAC, in another pair of modified let-2 reporter minigenes (M2) (Fig. 5A), and found that disruption of the stretch also phenocopied the asd-2 mutants (Fig. 5C). Furthermore, the M2 mutant reporter no longer responded to the overexpression of the ASD-2b cDNA (Fig. 5D). We also constructed a pair of minigenes (M3) in which a conserved UGCAUG element is disrupted (Fig. 5A), and found that the M3 mutant reporter responded to ASD-2b overexpression (Fig. 5E) as did the wild-type (WT) reporter (Fig. 4C). These results indicated that the QRE-like stretch is essential for ASD-2b to enforce expression of E10-RFP.

To examine whether ASD-2 directly binds to the QRElike stretch in vitro, we performed an electrophoretic mobility shift assay (EMSA) with RNA probes harboring the intact stretch or the mutation as in the M2 mutant reporter (Fig. 5F). The recombinant protein of the ASD-2 GSG/STAR domain (ASD-2 STAR) shifted the mobility 

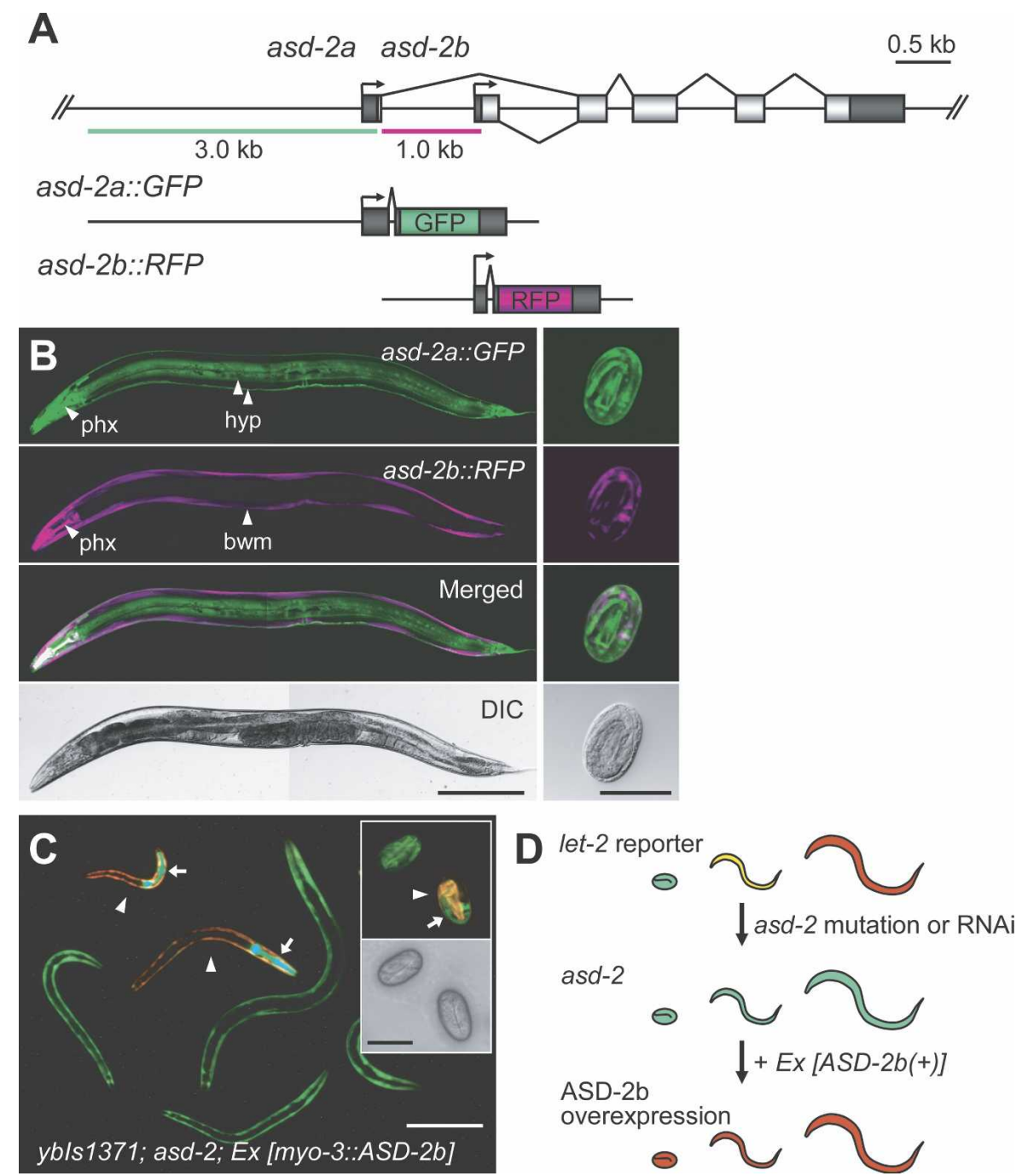

ASD-2b

overexpression

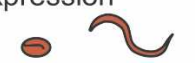

Figure 4. Expression and function of $a s d-2 b$ in the body wall muscles. $(A)$ Schematic representation of the asd-2 transcriptional fusion minigenes asd-2a::GFP and asd-2b::RFP. Green and magenta lines indicate the asd-2a promoter and the asd-2b promoter regions, respectively, used in the constructs. (B) Confocal images of the expression patterns of the asd-2 transcriptional fusions in an adult (left column) and a late embryo (right column). Expression of asd-2a::GFP (green) is detected in hypodermis (hyp) and pharyngeal muscles (phx). Expression of asd-2b::RFP (magenta) is detected in the body wall muscles (bwm) and the pharyngeal muscles (phx). (Bottom) A DIC image of the same field. Bars: left column, $100 \mu \mathrm{m}$; right column, $50 \mu \mathrm{m}$. (C) Microphotograph of the let-2 reporter allele ybIs1371 in the asd-2 (yb1423) mutant background carrying an extrachromosomal array for ASD-2b expression under the myo-3 promoter. Arrowheads indicate young larvae and an embryo (shown in inset) carrying the array. Arrows indicate the pharynges of transgenic worms expressing GFP from myo-2::GFP, a coinjected marker of transformation. Bars: $100 \mu$ m; inset, $50 \mu \mathrm{m}$. (D) Summary of the effects of asd-2 expression on the let-2 reporter.

of the wild-type RNA probe (WT) in a dose-dependent manner (Fig. 5F, lanes 2-6), but not of the M2 mutant probe (Fig. 5F, lanes 8-11), demonstrating the direct and specific binding of ASD-2 to the QRE-like stretch in vitro.

asd-2 promotes removal of let- 2 intron 10 in the late larval stages

Next, we examined the processing steps of the endogenous let-2 pre-mRNA. When we look at exon 8 to exon 11 of the let-2 pre-mRNA, mutually exclusive inclusion of exon 9 or exon 10 requires sequential removal of two flanking introns. As intron 9 (30 nucleotides) is too short to be excised due to steric interference between the donor and the possible branch site (Smith et al. 1993; Southby et al. 1999), we can expect four introns to be removed in the first splicing, and the subsequent second splicing to produce either of the two mature let-2 mRNAs (Fig. 6A). To gain insight into the processing course of the endogenous let-2 pre-mRNA, we tried to detect and compare the amounts of the four possible processing intermediates or partially spliced RNAs (Fig. 6A) in the synchronized wild-type and asd-2 mutant worms. 
Ohno et al.
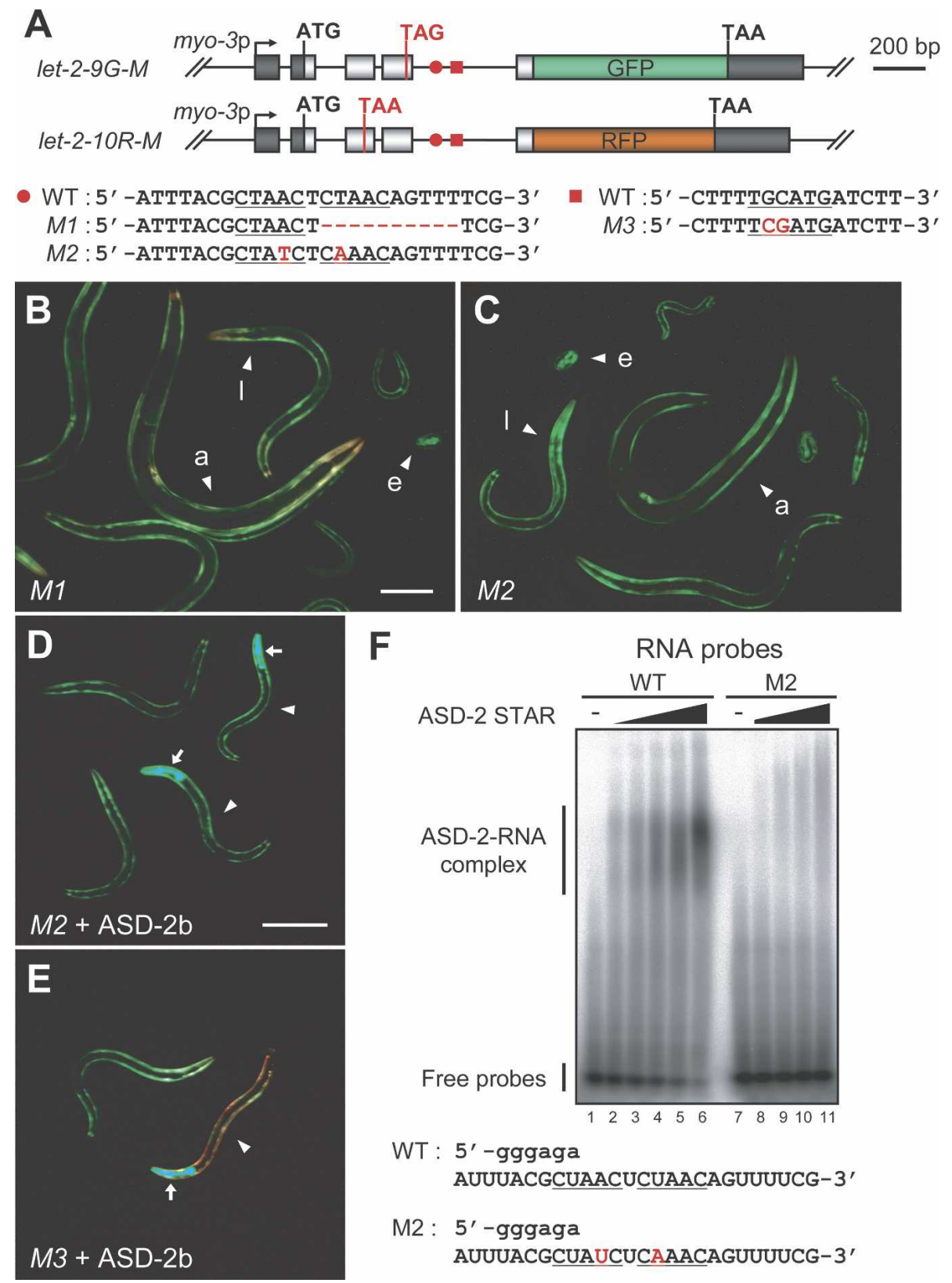

Figure 5. A regulatory element essential for the developmental switching of the let-2 alternative splicing by ASD-2b. (A) Schematic representation (top) and sequences (bottom) of the modified let-2 reporter minigenes $M 1, M 2$, and $M 3$. A red circle and a red square indicate the positions of the mutation sites for M1/M2 and $M 3$, respectively. Sequences fitting the consensus of QRE and a UGCAUG element are underlined. Mutated sequences are indicated in red. Dashed line indicates deletion. $(B, C)$ Transgenic worms expressing a pair of the let-2-M1 minigenes $(B)$ and a pair of the let-2-M2 minigenes (C). Arrowheads indicate an embryo (e), an L3 larva (1), and a young adult (a). Bar in $B, 100 \mu \mathrm{m} .(D, E)$ Transgenic worms overexpressing ASD-2b in body wall muscles, and carrying a pair of either the let-2-M2 minigenes $(D)$ or the let-2-M3 minigenes $(E)$. Arrowheads indicate young larvae carrying the extrachromosomal array for ASD-2b expression with a pharyngeal GFP marker (arrows). Bar in $D, 100 \mu \mathrm{m}$. (F) EMSA. Twofold dilution series of the recombinant ASD-2 STAR protein is used. Sequences of the wild-type and M2 probes are indicated at the bottom. The QRE-like sequences are underlined. Mutation sites are indicated in red.

To quantify the amounts of the let-2 processing intermediates, we prepared two pairs of PCR primers to amplify the complementary DNA fragments for the let-2 RNAs retaining intron(s). One primer set was designed on exon 8 and intron 10 to amplify partially spliced RNAs in which either of the upstream flanking introns was removed (Fig. 6B, left panel). The primer set amplified the unspliced pre-mRNA (8-9-10-11) and partially spliced RNAs in which intron 8 was excised (8/9-10-11) or the intron between exon 8 and exon 10 was excised (8/10-11). The other primer set was designed on intron 8 and exon 11 to amplify partially spliced RNAs in which 
A
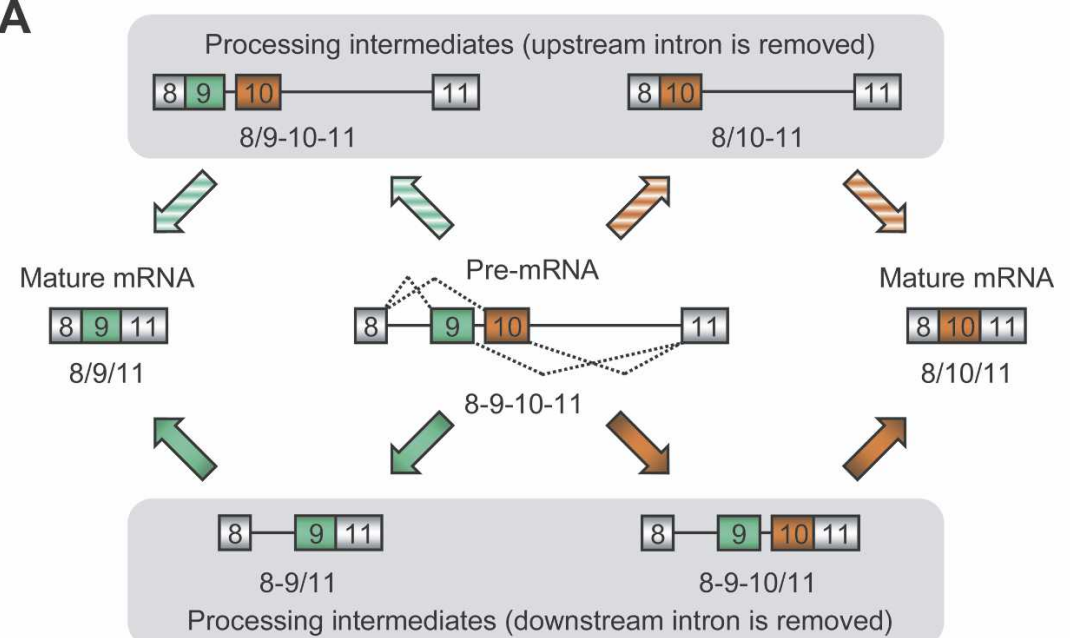

B
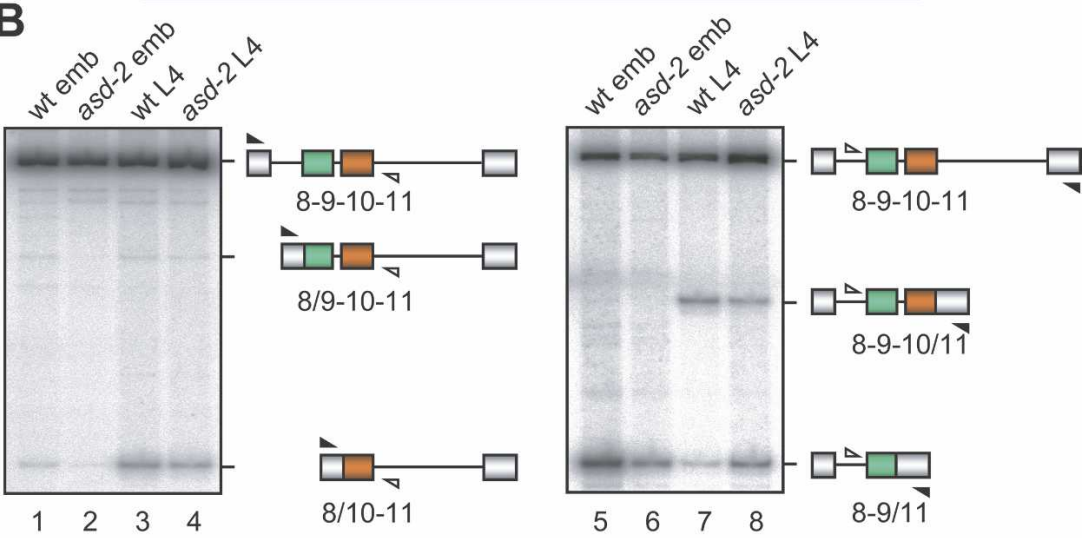

C

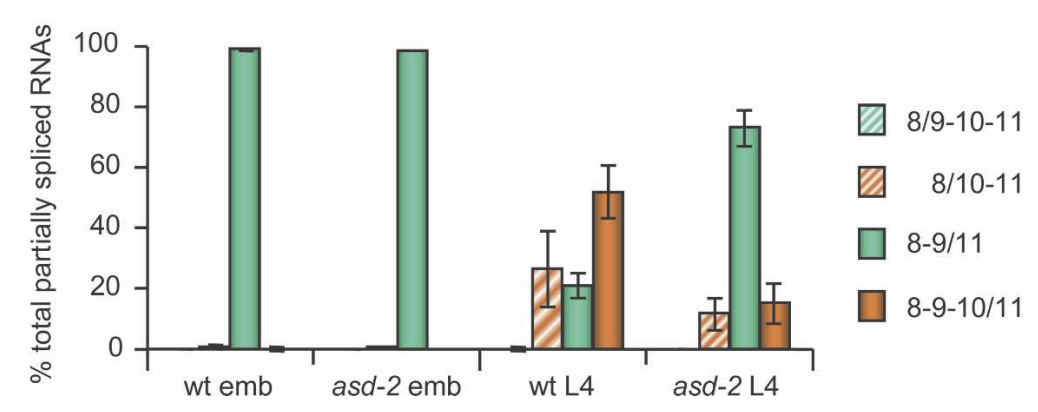

Figure 6. Analysis of partially spliced let-2 RNAs as the intermediates of the alternative pre-mRNA processing. (A) A model of twostep pre-mRNA processing ensuring the let-2 mutually exclusive alternative splicing. Four possible courses leading to the production of the two mature mRNAs are indicated. One of four introns (dotted lines) flanking the mutually exclusive exons is removed in the first splicing to generate one of the four possible processing intermediates (shaded in gray), and the remaining intron is removed in the second splicing. We assumed that intron 9 is too short to be excised. (B) Radioactive RTPCR detection of the partially spliced let-2 RNAs in the synchronized embryos (emb) and L4 larvae (L4) of the wild-type (wt) and the asd-2 (yb1423) mutant worms. Two pairs of the PCR primers were used to detect the let-2 RNAs in which either of the upstream introns was removed (left panel), and either of the downstream introns was removed (right panel). The structure of each PCR product was analyzed by cloning and sequencing, and is schematically indicated. Closed and open triangles indicate the positions of the exonic and the intronic primers, respectively. $(C)$ The relative amount of each of the partially spliced RNAs. The amount of each of the RNA species was calculated by normalization to the amount of the unspliced premRNA. $n=1$ for asd-2 embryos; $n=3$ for others. Error bars indicate standard error of mean (SEM). either of the downstream flanking introns was removed (Fig. 6B, right panel). The primer set amplified the premRNA (8-9-10-11) as well as partially spliced RNAs in which intron 10 was removed (8-9-10/11), or the intron between exon 9 and exon 11 was removed (8-9/11).

The normalized amounts of the four partially spliced RNAs are shown in Figure 6C. In both the wild-type and the asd-2 mutant embryos, the $8-9 / 11$ form, which is committed to the exon 9 mRNA isoform, was almost exclusively detected (Fig. 6B [lanes 1,2,5,6], C), consistent with the exclusive expression of the exon 9 mRNA in the embryos. In parallel with the developmental switching to the exon 10 mRNA isoform in the wildtype L4 larvae, we observed switching in the major form of the partially spliced RNAs from the $8-9 / 11$ form to the 8-9-10/11 form, one of two possible intermediates leading to the exon 10 mRNA (Fig. 6B [lane 7], C). On the other hand, the amount of the 8-9/11 form still predominated over that of the 8-9-10/11 form in the L4 larvae of the asd-2 mutant (Fig. 6B [lane 8], C), consistent with its switching-defective phenotype. These results indicated that asd-2 is required for selective removal of intron 10 in this stage. We also observed substantial increase in the amount of the 8/10-11 form in the L4 larvae independent of the asd-2 functions (Fig. 6B [lanes 3,4], C), which may contribute to the fraction of the exon 10 mRNA in the adult stage (Fig. 2B) and the color change of the let-2 reporter in the asd-2 senile adults.

The 8-9/11 form, the only intermediate detected in the embryos, will further be processed exclusively into 
Ohno et al.

the exon 9 mRNA (Fig. 6A). However, the 8-9-10/11 form, the major intermediate in the wild-type L4 larvae, has two remaining splice acceptors, for exon 9 and exon 10 , and may not necessarily be processed into the exon 10 mRNA. We therefore analyzed the fate of a premRNA from a modified let-2 reporter minigene that carried only exon 8 to exon 10, and observed a selective use of the exon 10 splice acceptor (Fig. 7A [top panel], B [lane 1]). We reasoned that the selective use of the exon 10 acceptor (TTTTTAG) was due to the relative weakness of the exon 9 splice acceptor (ATTCTAG), since the exon 9 acceptor became predominantly selected (Fig. 7A [bottom panel], B [lane 2]) when the reporter was further modified, so that the exon 9 acceptor matched the consensus of U2AF65 binding for C. elegans (TTTTCAG) (Hollins et al. 2005). These results are schematically summarized in Figure $7 \mathrm{C}$.

\section{Discussion}

In the present study, we isolated asd-2 mutants defective in the switching of the developmentally regulated mutually exclusive alternative exons in the let-2 mRNAs, and compared the amounts of the intermediates of the post-transcriptional let-2 pre-mRNA processing between the wild-type and the asd-2 mutant.

Course of mutually exclusive alternative splicing of let-2 pre-mRNA and the switching mechanism by $A S D-2$

The unique property of the developmental switching and the isolation of the mutants of the let-2 alternative splicing allowed us to analyze the relative amounts of the partially spliced RNAs (Fig. 6). In the case of Nova-2regulated cassette exons in mice, Ule et al. (2006) ob-

A

B
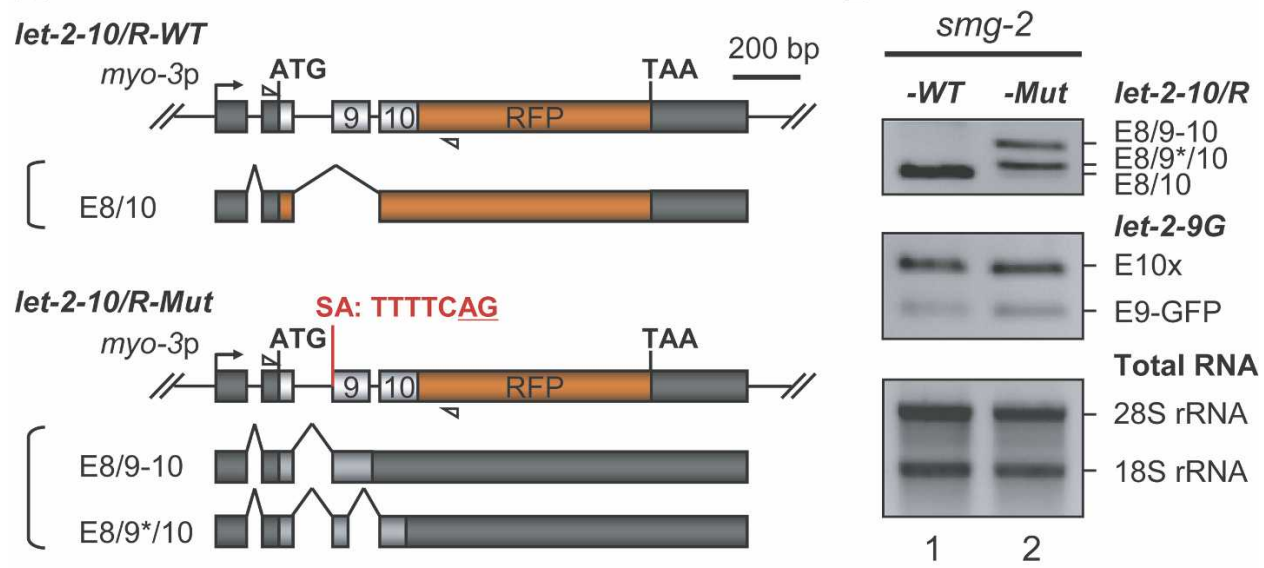

C

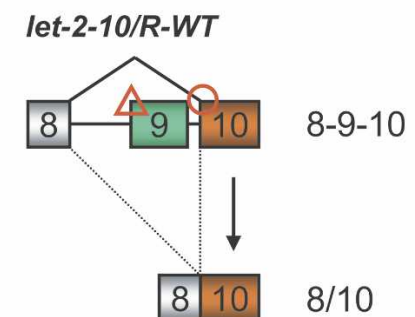

E9 SA $<$ E10SA
let-2-10/R-Mut
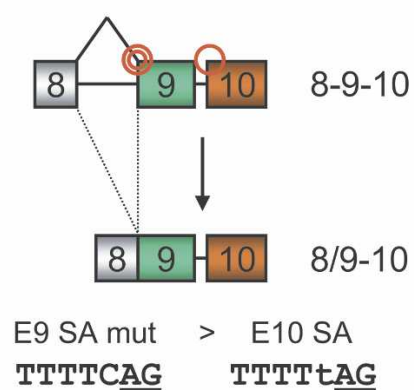

Figure 7. Biased selection between the splice acceptors for exon 9 and exon 10. (A) Structure of the let-2 reporter minigenes let-2-10/R-WT (top) and let-2-10/R-Mut (bottom), and mRNAs derived from the minigenes. The let-2 genomic DNA fragment spanning from exon 8 to exon 10 is connected in-frame to RFP cDNA. The splice acceptor (SA) for exon 9 (aTTctAG) is mutagenized to TTTTCAG in let-2-10/R-Mut. Structure of the mRNAs was confirmed by cloning and sequencing the RT-PCR products. Predicted ORFs are colored in red (E8/10-RFP) or gray (E8/9-10 and E8/9*/10). A cryptic donor site within exon 9 is used in the E8/9*/10 form. Open triangles indicate positions and directions of the PCR primers used to amplify the cDNAs in $B$. $(B)$ RT-PCR analysis of the mRNAs derived from the let-2-10/R minigenes $(t o p)$ and coinjected let-2-9G (middle). (Bottom) Total RNAs prepared from mixed-stage population. (Lane 1) let-2-10/R-WT. (Lane 2) let-2-10/R-Mut. Either of the let-2-10/R minigenes was coinjected with let-2-9G (Fig. 1B) into the smg-2 (yb979) mutant (Kuroyanagi et al. 2007), defective in NMD, to avoid degradation of the nonproductive mRNAs. Note that the E8/10-RFP form was exclusively produced from let-2-10/R-WT, while the E8/9-10 and E8/9*/10 forms were preferentially produced from let-2-10/R-Mut. (C) Schematic models of the biased selection of the exon 10 acceptor in let-2-10/R-WT pre-mRNA (left panel) and the exon 9 acceptor in let-2-10/R-Mut pre-mRNA (right panel). Note that the relatively stronger acceptor is selected in each case. 
served biased accumulation of a partially spliced RNA in which either of the upstream or the downstream flanking introns was removed in the exon-including condition, either in the wild-type or the Nova-2 knock-out brain. In general, the steady-state amount of the processing intermediate is determined by the rate of production (removal of an intron in the first splicing) and the rate of degradation (mainly the subsequent splicing), and therefore it does not necessarily reflect the rate of production of the mature mRNA isoform produced from the intermediate. Nevertheless, we found a dramatic switching in the major species of the partially spliced let-2 RNAs during larval development in parallel with the switching from the exon 9 to the exon 10 isoforms of the mature let-2 mRNA (Fig. 6C), suggesting that the major species of the partially spliced RNAs is the processing intermediate of the major course of the pre-mRNA processing. Furthermore, we observed a defect in the switching in the major species of the partially spliced let-2 RNAs in L4 larvae of the asd-2 mutant (Fig. 6C), consistent with the defect of the asd-2 mutant to produce the exon 10 mRNA. Assuming that asd-2 mutation does not affect the rate of the second splicing, the result indicates that asd-2 is required for the production of the major 8-9-10/ 11 form in the wild-type L4 larvae, and strongly suggests that the 8-9-10/11 form is the intermediate in the course of the let-2 pre-mRNA processing by which most of the exon 10 mRNA is produced in the later stages.

Figure 8 illustrates a model of the processing courses of the let-2 pre-mRNA and the mode of switching regulation by ASD-2 raised by the present study. The unique feature of the let-2 alternative splicing is that either of the two introns downstream from the mutually exclusive exons is removed in the first splicing out of four possible flanking introns (Fig. 6A). In the embryonic stage, or when asd-2 is inactive (Fig. 8, left panel), the splice donor of exon 9 (Fig. 8, green circle, GTACTG) is preferentially selected to produce the $8-9 / 11$ intermediate, since the splice donor of exon 10 (Fig. 8, green triangle, GCAAGA) is noncanonical and weaker (Farrer et al. 2002; Kabat et al. 2006), leading to the production of
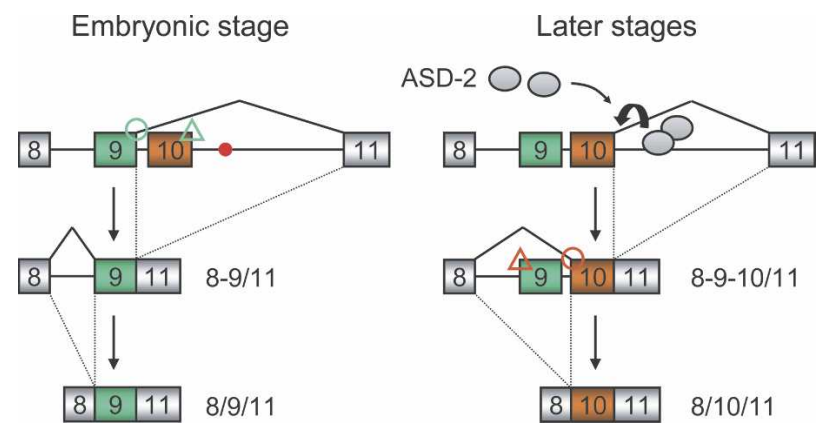

Figure 8. Models of the sequences of the let-2 alternative splicing and the mechanism of exon switching by ASD-2. (Left panel) A model of exon 9 selection in the embryonic stage or when asd-2 is inactive. (Right panel) A model of exon 10 selection when asd-2 is active in the late larval stages. Red dot indicates the ASD-2-binding site. See the text for detail. the exon 9 mRNA. On the other hand, when asd-2 is active in the late larval stages (Fig. 8, right panel), ASD-2 binds to the QRE-like cis-acting element in intron 10 to promote the selection of the weak exon 10 splice donor, leading to the production of the 8-9-10/11 intermediate. Thereafter, the splice acceptor of exon 10 (Fig. 8, red circle, TTTTTAG) is preferentially selected, as that of exon 9 (Fig. 8, red triangle, ATTCT $\underline{\text { AG) }}$ is weaker.

The model described above indicates that the fate of the pre-mRNA, whether to be the exon 9 or exon 10 mRNA, is primarily determined by the removal of a flanking intron in the first splicing. The model also indicates that the mutually exclusive nature of the let-2 alternative exons is ensured by the ordered removal of the flanking introns and the asymmetric strength of the splice donors and the acceptors of the two alternative exons, as well as by the steric interference of the two exons. In the present study, we observed that endogenous $a s d-2 b$ was expressed in embryos (Fig. $4 \mathrm{~B}$ ) and that the let-2 exon 10 mRNA isoform was eventually produced in the adult stage, even in the asd-2 mutant background. These observations suggest a possibility that besides ASD-2, other regulators, such as modulators of the ASD-2 function and/or other trans-factors, are involved in the let-2 alternative splicing. Regulation of the order of the intron removal may also be involved in the developmental switching as implicated by the substantial increase in the 8/10-11 intermediate in the L4 stage (Fig. 6C). It would be an interesting challenge to elucidate all of the factors involved in the precise control of developmental switching of the let-2 mutually exclusive alternative splicing in vivo.

\section{STAR family RNA-binding protein ASD-2b regulates alternative splicing in $\mathrm{C}$. elegans}

STAR family proteins are known as key regulators of the various aspects of biological processes through multiple functions in the RNA metabolism. In the STAR family, the GLD-1 subfamily has been shown to play multiple roles through direct binding to the target RNAs. The Quaking gene generates three isoforms of proteins by alternative splicing. The nuclear isoform QKI-5 regulates alternative splicing of the myelin-associated glycoprotein (MAG) gene (Wu et al. 2002), while the balance of the nuclear and cytoplasmic isoforms QKI-6 and QKI-7 regulates the nuclear export of the myelin basic protein (MBP) mRNA (Larocque et al. 2002). Drosophila how encodes two alternative isoforms and the balance between the opposing activities of the two isoforms regulates tendon cell differentiation through the level of the transcription factor Stripe (Nabel-Rosen et al. 1999, 2002). Unlike vertebrates and insects, C. elegans has two members of the GLD-1 subfamily of genes. The cytoplasmic protein GLD-1 (Jones et al. 1996) regulates the germ cell development and sexual identity by regulating mRNA translation and stability (Jan et al. 1999; Lee and Schedl 2001, 2004; Schumacher et al. 2005). Our genetic studies demonstrate that ASD-2b regulates alternative splicing in the body wall muscles. Although the molecu- 
lar function of the ASD-2a isoform should be characterized further, ASD-2a may play essential roles in the nematode development, since the asd-2 deletion allele ok483, in which common exon 3 and exon 4 are deleted, is lethal (WormBase, http://www.wormbase.org), while the $a s d-2 b$-specific mutants look otherwise normal and only show the color phenotype. The multiple nuclear and cytoplasmic isoforms produced by alternative splicing and/or gene duplication may have conferred the multiple functions in mRNA metabolisms to the subfamily.

Sam68 and its paralogs, SLM1 and SLM2 of mammals constitute of the Sam68/SLM subfamily in the STAR family (Fig. 3B; Di Fruscio et al. 1999). The subfamily has been shown to mediate the signal transduction to RNA metabolism by modulating the RNA-binding properties upon phosphorylation (Stoss et al. 2001; Matter et al. 2002; Haegebarth et al. 2004; Paronetto et al. 2007). Although signals regulating the GLD-1 subfamily are to be elucidated, regulation of the RNA-binding property may be involved in the regulation of the let-2 alternative splicing by ASD-2b in vivo.

\section{RNA-binding specificity of ASD-2}

We identified the essential QRE-like cis-element for ASD-2b to regulate let-2 alternative splicing. Several studies have identified the direct binding sites of the GLD-1 subfamily and addressed the consensus of the binding sequences. Ryder et al. (2004) demonstrated that GLD-1 preforms a dimer, and specifically binds to the UACU/C/A)A stretch(es) in the target mRNAs. They also showed that QKI recognizes the consensus $\mathrm{NA}(\mathrm{A}>\mathrm{C})$ UAA by extensive mutagenesis of the GLD-1 consensus sequence (Ryder and Williamson 2004). Enrichment of the high-affinity RNA ligands using systematic evolution of ligands by exponential enrichment (SELEX) defined the QRE as a bipartite consensus sequence NACUAAY-N ${ }_{1-20}$-UAAY, consisting of a core site (NACUAAY) and a tetra-nucleotide half site (UAAY) (Galarneau and Richard 2005). Recently, the primary sequence and the secondary structure requirement for Drosophila HOW response element (HRE) has been determined as the ACUAA stretch in the loop (Israeli et al. 2007). The results from these studies indicate that the GSG/STAR domains of the GLD-1 subfamily share not only the high amino acid sequence identity, but also the consensus binding sequence determined as QRE, although the sequence that binds with the highest affinity may differ among the GLD-1 subfamily members (Saccomanno et al. 1999; Ryder and Williamson 2004).

The cis-element we identified in this study consists of direct repeats of the penta-nucleotide stretch, CUAAC, which matches five out of six nucleotides of the QRE core site. The tetra-nucleotide QRE half site, which can be either upstream of or downstream from the core site, has been shown to be required for the high-affinity binding (Galarneau and Richard 2005) and the requirement of the half site in the proximity has also been suggested for GLD-1 (Ryder et al. 2004). It is likely that the direct repeats of the penta-nucleotide compensated the require- ment of the complete core site for let-2 regulation by ASD-2 as in the case of QRE-1 in the 3'-untranslated region (UTR) of MBP mRNA, where multiple clusters of the half site constitute QRE /Galarneau and Richard 2005). QKI and GLD-1 form homodimers via the QUA1 domain (Chen and Richard 1998; Ryder et al. 2004) and the E48 residue of QKI, conserved among the GLD-1 subfamily proteins (Fig. 3A), is essential for the dimer formation and the function of the Quaking gene /Chen and Richard 1998). We identified a substitution of a conserved residue in the QUA1 domain in the asd-2 (yb1422) mutant (Fig. 3A), suggesting the functional importance of the QUA1 domain for the ASD-2 function. ASD-2 may also form a homodimer via the conserved QUA1 domain, and each subunit may contact one CUAAC stretch in binding to the let-2 pre-mRNA. Comparison of the dimer formation activities and the RNAbinding properties of the GSG/STAR domains derived from the various asd-2 mutants isolated in this study would lead to further understanding of the RNA-recognition by the STAR family proteins.

Recent global and comparative analyses of the alternatively spliced exons have demonstrated that the (A)CTAAC stretch, which resembles the QRE, is enriched in the introns flanking the alternative exons in both mammals and nematodes, suggesting that the stretch is an intronic alternative splicing regulator (Kabat et al. 2006; Sugnet et al. 2006; Das et al. 2007; Yeo et al. 2007). The GLD-1 subfamily may be involved in the regulation of these genes, although the requirement of the (A)CTAAC stretch and the function of the GLD-1 subfamily are to be experimentally addressed.

\section{Visualization of the alternative splicing patterns in vivo}

In the present study, we utilized a pair of symmetric minigenes, both of which contain four exons and three intervening introns for visualizing the expression profiles of the let-2 mutually exclusive alternative exons (Fig. 1B). In contrast, for visualizing the tissue-specific expression profiles of the egl-15 mutually exclusive exons, we utilized a single unified minigene, in which GFP and RFP cDNAs were introduced into the alternative exons (Kuroyanagi et al. 2006). With the unified reporter minigene, we demonstrated that mutually exclusive selection of the egl-15 alternative exons is regulated by the upstream-most intron, and that the downstream-most intron is dispensable for the regulation (Kuroyanagi et al. 2006). In general, the downstream flanking intron and the downstream constitutive exon should be included in the alternative splicing reporter minigenes, since the ciselement(s) in the intron downstream from the alternative exon(s) could be essential for the regulation, as demonstrated in this study. As the let-2 reporter minigenes are symmetric and contain the intact genomic sequence, except for a modification in one exon in each of the reporter minigenes, we can assume that expression of the reporter reflects the regulation of the endogenous gene, and that the simultaneous modification of the cis-ele- 
ment(s) in both of the minigene pairs should cause the same effect on the pre-mRNAs derived from these minigenes, as demonstrated in this study. We therefore conclude that a symmetric pair of minigenes is feasible for the visualization of the alternative splicing patterns and for the elucidation of the regulation mechanisms in $C$. elegans.

The utilization of dichromatic alternative splicing reporters has recently been described in other organisms. Carstens and colleagues (Newman et al. 2006) utilized a pair of reporter minigenes for the FGFR2 mutually exclusive exons IIIb and IIIc with a similar structure to the let-2 reporter. They demonstrated that the reporter and a fluorescence-activated cell sorter (FACS) facilitated the analysis of the function of various trans-factors in the selection of the mutually exclusive exons. Cooper and colleagues (Orengo et al. 2006) utilized another type of a single dichromatic reporter minigene, in which GFP and RFP cDNAs were fused in the different frame so that mutually exclusive expression of GFP and RFP from the single minigene reflected the expression of the alternative isoforms. Garcia-Blanco and colleagues (Bonano et al. 2006) generated knock-in mice that visualized the repression of the FGFR2 exon IIIb in vivo. The mice carry an RFP-minigene to monitor efficiency of constitutive splicing and a GFP-minigene to monitor repression of FGFR2 exon IIIb at a single-cell resolution in vivo. The advantage of these dichromatic fluorescent alternative splicing reporters, including those in our study, is that relative abundance of the two alternative isoforms in each cell can be reliably analyzed with a minimal effect of other aspects of gene expression such as a transcription level. Application of these alternative splicing reporters will facilitate expression profiling and experimental identification of trans-factors and cis-elements of various alternative splicing events.

In conclusion, visualization of the expression profiles of the alternative exons enabled us to isolate the splicing mutants and identify trans-acting factors and cis-acting elements. Isolation of the splicing mutants enabled us to detect and analyze the partially spliced pre-mRNAs or processing intermediates. These experimental data led to a model of how only one out of two exons is exclusively included in each mRNA and how the factor and elements regulate the fate of the exons. Experimental approaches of visualization of the splicing patterns, genetic analyses of splicing mutants, and detection of the processing intermediates in vivo, together with the comprehensive bioinformatic approaches, would open the way to further elucidate the spatiotemporally regulated fate of pre-mRNAs, or deciphering the splicing codes in metazoans.

\section{Materials and methods}

\section{Plasmid construction}

We constructed the reporter minigenes essentially as described in the text. To construct the reporter cassettes for let-2-9G, let-2-10R, and let-2-10/R-WT, we amplified the let-2 genomic
DNA fragments spanning from exon 8 to either exon 11 or exon 10 and cloned them into Gateway Entry vectors (Invitrogen) carrying either EGFP (Clontech) or mRFP1 (Campbell et al. 2002) cDNAs using the In-Fusion system (BD Biosciences). We introduced termination codons by using QuickChange (Stratagene). To construct the ASD-2b Entry vector, we amplified the ASD-2b cDNA fragment and cloned it in pENTR-D/TOPO (Invitrogen). We constructed the Expression vectors by homologous recombination between the reporter cassettes and the myo-3 promoter vector pDEST-myo-3p (Kuroyanagi et al. 2006) by utilizing the Gateway system (Invitrogen). We generated M1, $M 2$, and $M 3$ mutant minigenes from myo-3::1et-2-9G and myo$3:: 1 e t-2-10 R$, and myo-3::1et-2-10/R-Mut from myo-3::1et-2-10/ $R-W T$ by mutagenesis utilizing QuickChange (Stratagene). We amplified the genomic fragments of the $a s d-2 a$ and $a s d-2 b$ promoter regions and cloned them into promoterless Expression vectors to construct asd-2a::GFP and asd-2b::RFP. We confirmed all of the constructs by sequence analysis. Sequences of the primers used in the construction are available in the Supplemental Material.

\section{Worm culture and microscopy}

We cultured worms following standard methods (Brenner 1974). We prepared the transgenic lines essentially as described (Mello et al. 1991) using lin-15 (n765) as a host. We generated integrant lines by ultraviolet light irradiation (Mitani 1995). We treated ybIs1371 [myo-3::1et-2-9G myo-3::let-2-10R lin-15(+)] animals with $47 \mathrm{mM}$ ethyl methanesulfonate (EMS) for $4 \mathrm{~h}$ at room temperature, and we screened the $\mathrm{F} 2$ progeny for mutants with altered expression profiles of the GFP and RFP under a fluorescence stereoscope (MZ16FA; Leica) equipped with a dual-bandpass filter set. We captured the images of the let-2 reporter worms with CCD cameras (E300 and DP71; Olympus). We used a confocal microscope (Fluoview FV500; Olympus) for image scanning and processed the acquired images with Metamorph (Molecular Devices). We identified the asd-2 gene by SNP-based mapping essentially as described (Wicks et al. 2001). Briefly, we crossed the mutants with the Hawaiian wild-type strain, CB4856, which has many SNPs compared with the standard wild-type strain N2, and the SNPs of each F2 progeny with the green phenotype were analyzed by either restriction fragment length polymorphisms or direct sequencing of the PCR-amplified genomic fragments. Of $\sim 75$ predicted genes within the defined 391-kb region, we amplified and sequenced 13 RNA-related genes. We performed RNAi by soaking $\mathrm{L} 4$ hermaphrodites as described previously (Kuroyanagi et al. 2000; Maeda et al. 2001) by using dsRNAs corresponding to nucleotides 164-841 of the ASD-2b ORF.

\section{$R T-P C R$}

We prepared total RNAs and performed RT-PCR analysis essentially as described previously (Kuroyanagi et al. 2006). We digested the RT-PCR products for the let-2 reporter and the endogenous let-2 mRNAs with BglII before electrophoresis separation. Sequences of the primers used in the RT-PCR assays are available in the Supplemental Material.

\section{EMSA}

We prepared the recombinant protein for the GSG/STAR domain of ASD-2 (ASD-2 STAR), corresponding to amino acids 55-280 of ASD-2b, as a His-tagged protein. We introduced the ASD-2 STAR cDNA fragment into the modified bacterial coldinduction vector (Kuroyanagi et al. 2007) and induced the pro- 
tein production following the standard cold-induction procedure (Takara). We purified the His-ASD-2 STAR protein with Ni-NTA agarose (Qiagen). We prepared the ${ }^{32} \mathrm{P}$-labeled wildtype and M2 RNA probes by in vitro transcription with $\left[\alpha^{32}\right.$ P]UTP and T7 RNA polymerase (Takara). We incubated the gel-purified RNA probes alone or with increasing concentrations of His-ASD-2 STAR in $25 \mu \mathrm{L}$ of RNA-binding buffer $(20$ $\mathrm{mM}$ HEPES-KOH at $\mathrm{pH} 7.9,320 \mathrm{mM} \mathrm{KCl}, 5 \%$ glycerol, $1 \%$ Triton X-100, $1 \mathrm{mM}$ DTT, $0.1 \mathrm{mM}$ PMSF) supplemented with $130 \mathrm{ng} / \mu \mathrm{L}$ Escherichia coli tRNA and $50 \mathrm{ng} / \mu \mathrm{L}$ BSA for $30 \mathrm{~min}$ at $20^{\circ} \mathrm{C}$. Each sample was separated on a nondenaturing polyacrylamide gel and visualized with a phosphorimage analyzer (FLA-3000G; Fuji Film).

\section{Analysis of partially spliced let-2 RNAs}

We performed RT with random hexamers. We performed PCR following standard protocols supplemented with $\left[\alpha^{32} \mathrm{P}\right] \mathrm{dCTP}$, and separated the PCR products on $5 \%$ nondenaturing polyacrylamide gels and quantified the signal intensity of each band with a phosphorimage analyzer (FLA-3000G; Fuji Film). We calculated the amount of each processing intermediate by normalization to that of the pre-mRNA in the same reaction, taking their $\mathrm{G}+\mathrm{C}$ contents into account. We confirmed the identity of each band by cloning and sequence analysis. We performed control PCRs using RT products without reverse transcriptase (shown in Supplemental Fig. 1) to calculate the contamination of genomic DNAs in the total RNA fraction. Sequences of the primers used in the PCRs are available in the Supplemental Material.

\section{Database information}

The gene assignment asd-2 has been approved by the CGC Genetic Map and Nomenclature Curator. The nucleotide sequences of the asd-2 cDNAs have been deposited in the GenBank/EMBL/DDBJ databases with the accession numbers EF630625 and EF630626.

\section{Acknowledgments}

We thank S. Mitani at Tokyo Women's Medical University for assistance in the mutant screening. We thank T. Nojima, A. Takeuchi, Y. Ogawa, and N. Kataoka at TMDU for discussions and comments on the manuscript. We thank R.Y. Tsien at University of California at San Diego and the Caenorhabditis Genetics Center for materials. We thank Y. Kikuchi, Y. Usami, and M. Hagiwara for technical assistance. This work was supported by a Grant-in-Aid for Scientific Research on Priority Areas from the Ministry of Education, Culture, Sports, Science, and Technology of Japan (to H.K. and M.H.). G.O. contributed to overall experiments. M.H. and H.K. organized this work. We all discussed the results and wrote the manuscript.

\section{Reference}

Baraniak, A.P., Lasda, E.L., Wagner, E.J., and Garcia-Blanco, M.A. 2003. A stem structure in fibroblast growth factor receptor 2 transcripts mediates cell-type-specific splicing by approximating intronic control elements. Mol. Cell. Biol. 23: 9327-9337.

Baraniak, A.P., Chen, J.R., and Garcia-Blanco, M.A. 2006. Fox-2 mediates epithelial cell-specific fibroblast growth factor receptor 2 exon choice. Mol. Cell. Biol. 26: 1209-1222.

Black, D.L. 2003. Mechanisms of alternative pre-messenger
RNA splicing. Annu. Rev. Biochem. 72: 291-336.

Blencowe, B.J. 2006. Alternative splicing: New insights from global analyses. Cell 126: 37-47.

Bonano, V.I., Oltean, S., Brazas, R.M., and Garcia-Blanco, M.A. 2006. Imaging the alternative silencing of FGFR2 exon IIIb in vivo. RNA 12: 2073-2079.

Brenner, S. 1974. The genetics of Caenorhabditis elegans. Genetics 77: 71-94.

Campbell, R.E., Tour, O., Palmer, A.E., Steinbach, P.A., Baird, G.S., Zacharias, D.A., and Tsien, R.Y. 2002. A monomeric red fluorescent protein. Proc. Nat1. Acad. Sci. 99: 7877-7882.

Chen, T. and Richard, S. 1998. Structure-function analysis of Qk1: A lethal point mutation in mouse quaking prevents homodimerization. Mol. Cell. Biol. 18: 4863-4871.

Chen, C.D., Kobayashi, R., and Helfman, D.M. 1999. Binding of hnRNP $\mathrm{H}$ to an exonic splicing silencer is involved in the regulation of alternative splicing of the rat $\beta$-tropomyosin gene. Genes \& Dev. 13: 593-606.

Crawford, J.B. and Patton, J.G. 2006. Activation of $\alpha$-tropomyosin exon 2 is regulated by the SR protein 9G8 and heterogeneous nuclear ribonucleoproteins $\mathrm{H}$ and F. Mol. Cell. Biol. 26: 8791-8802.

Das, D., Clark, T.A., Schweitzer, A., Yamamoto, M., Marr, H., Arribere, J., Minovitsky, S., Poliakov, A., Dubchak, I., Blume, J.E., et al. 2007. A correlation with exon expression approach to identify cis-regulatory elements for tissue-specific alternative splicing. Nucleic Acids Res. 35: 4845-4857.

Di Fruscio, M., Chen, T., and Richard, S. 1999. Characterization of Sam68-like mammalian proteins SLM-1 and SLM-2: SLM-1 is a Src substrate during mitosis. Proc. Natl. Acad. Sci. 96: 2710-2715.

Ebersole, T.A., Chen, Q., Justice, M.J., and Artzt, K. 1996. The quaking gene product necessary in embryogenesis and myelination combines features of RNA binding and signal transduction proteins. Nat. Genet. 12: 260-265.

Expert-Bezancon, A., Sureau, A., Durosay, P., Salesse, R., Groeneveld, H., Lecaer, J.P., and Marie, J. 2004. hnRNP A1 and the SR proteins ASF/SF2 and SC35 have antagonistic functions in splicing of $\beta$-tropomyosin exon 6B. J. Biol. Chem. 279: 38249-38259.

Farrer, T., Roller, A.B., Kent, W.J., and Zahler, A.M. 2002. Analysis of the role of Caenorhabditis elegans GC-AG introns in regulated splicing. Nucleic Acids Res. 30: 33603367.

Faustino, N.A. and Cooper, T.A. 2003. Pre-mRNA splicing and human disease. Genes \& Dev. 17: 419-437.

Francis, R., Barton, M.K., Kimble, J., and Schedl, T. 1995. gld-1, a tumor suppressor gene required for oocyte development in Caenorhabditis elegans. Genetics 139: 579-606.

Galarneau, A. and Richard, S. 2005. Target RNA motif and target mRNAs of the Quaking STAR protein. Nat. Struct. Mol. Biol. 12: 691-698.

Graham, P.L., Johnson, J.J., Wang, S., Sibley, M.H., Gupta, M.C., and Kramer, J.M. 1997. Type IV collagen is detectable in most, but not all, basement membranes of Caenorhabditis elegans and assembles on tissues that do not express it. $J$. Cell Biol. 137: 1171-1183.

Graveley, B.R. 2005. Mutually exclusive splicing of the insect Dscam pre-mRNA directed by competing intronic RNA secondary structures. Cell 123: 65-73.

Gromak, N., Matlin, A.J., Cooper, T.A., and Smith, C.W. 2003 a. Antagonistic regulation of $\alpha$-actinin alternative splicing by CELF proteins and polypyrimidine tract binding protein. RNA 9: 443-456.

Gromak, N., Rideau, A., Southby, J., Scadden, A.D., Gooding, C., Huttelmaier, S., Singer, R.H., and Smith, C.W. 2003b. 
The PTB interacting protein raver1 regulates $\alpha$-tropomyosin alternative splicing. EMBO J. 22: 6356-6364.

Haegebarth, A., Heap, D., Bie, W., Derry, J.J., Richard, S., and Tyner, A.L. 2004. The nuclear tyrosine kinase BRK/Sik phosphorylates and inhibits the RNA-binding activities of the Sam68-like mammalian proteins SLM-1 and SLM-2. J. Biol. Chem. 279: 54398-54404.

Hollins, C., Zorio, D.A., MacMorris, M., and Blumenthal, T. 2005. U2AF binding selects for the high conservation of the C. elegans 3' splice site. RNA 11: 248-253.

Israeli, D., Nir, R., and Volk, T. 2007. Dissection of the target specificity of the RNA-binding protein HOW reveals $d p p$ mRNA as a novel HOW target. Development 134: 21072114.

Jan, E., Motzny, C.K., Graves, L.E., and Goodwin, E.B. 1999. The STAR protein, GLD-1, is a translational regulator of sexual identity in Caenorhabditis elegans. EMBO J. 18: 258-269.

Jin, Y., Suzuki, H., Maegawa, S., Endo, H., Sugano, S., Hashimoto, K., Yasuda, K., and Inoue, K. 2003. A vertebrate RNAbinding protein Fox-1 regulates tissue-specific splicing via the pentanucleotide GCAUG. EMBO J. 22: 905-912.

Johnson, J.M., Castle, J., Garrett-Engele, P., Kan, Z., Loerch, P.M., Armour, C.D., Santos, R., Schadt, E.E., Stoughton, R., and Shoemaker, D.D. 2003. Genome-wide survey of human alternative pre-mRNA splicing with exon junction microarrays. Science 302: 2141-2144.

Jones, A.R. and Schedl, T. 1995. Mutations in gld-1, a female germ cell-specific tumor suppressor gene in Caenorhabditis elegans, affect a conserved domain also found in Src-associated protein Sam68. Genes \& Dev. 9: 1491-1504.

Jones, A.R., Francis, R., and Schedl, T. 1996. GLD-1, a cytoplasmic protein essential for oocyte differentiation, shows stageand sex-specific expression during Caenorhabditis elegans germline development. Dev. Biol. 180: 165-183.

Jones, R.B., Carstens, R.P., Luo, Y., and McKeehan, W.L. 2001 a. $5^{\prime}$ - and $3^{\prime}$-terminal nucleotides in the FGFR2 ISAR splicing element core have overlapping roles in exon IIIb activation and exon IIIc repression. Nucleic Acids Res. 29: 3557-3565.

Jones, R.B., Wang, F., Luo, Y., Yu, C., Jin, C., Suzuki, T., Kan, M., and McKeehan, W.L. 2001b. The nonsense-mediated decay pathway and mutually exclusive expression of alternatively spliced FGFR2IIIb and -IIIc mRNAs. J. Biol. Chem. 276: 4158-4167.

Kabat, J.L., Barberan-Soler, S., McKenna, P., Clawson, H., Farrer, T., and Zahler, A.M. 2006. Intronic alternative splicing regulators identified by comparative genomics in nematodes. PLoS Comput. Biol. 2: e86. doi: 10.1371/journal.pcbi. 0020086.

Kramer, A. and Utans, U. 1991. Three protein factors (SF1, SF3 and U2AF) function in pre-splicing complex formation in addition to snRNPs. EMBO T. 10: 1503-1509.

Kuroyanagi, H., Kimura, T., Wada, K., Hisamoto, N., Matsumoto, K., and Hagiwara, M. 2000. SPK-1, a C. elegans SR protein kinase homologue, is essential for embryogenesis and required for germline development. Mech. Dev. 99: 5164.

Kuroyanagi, H., Kobayashi, T., Mitani, S., and Hagiwara, M. 2006. Transgenic alternative-splicing reporters reveal tissuespecific expression profiles and regulation mechanisms in vivo. Nat. Methods 3: 909-915.

Kuroyanagi, H., Ohno, G., Mitani, S., and Hagiwara, M. 2007. Fox-1 family and SUP-12 coordinately regulate tissue-specific alternative splicing in vivo. Mol. Cell. Biol. 27: 86128621.

Larocque, D., Pilotte, J., Chen, T., Cloutier, F., Massie, B., Pedraza, L., Couture, R., Lasko, P., Almazan, G., and Richard,
S. 2002. Nuclear retention of MBP mRNAs in the quaking viable mice. Neuron 36: 815-829.

Lee, M.H. and Schedl, T. 2001. Identification of in vivo mRNA targets of GLD-1, a maxi-KH motif containing protein required for C. elegans germ cell development. Genes \& Dev. 15: $2408-2420$.

Lee, M.H. and Schedl, T. 2004. Translation repression by GLD-1 protects its mRNA targets from nonsense-mediated mRNA decay in C. elegans. Genes \& Dev. 18: 1047-1059.

Lehmann-Blount, K.A. and Williamson, J.R. 2005. Shape-specific nucleotide binding of single-stranded RNA by the GLD-1 STAR domain. J. Mol. Biol. 346: 91-104.

Letunic, I., Copley, R.R., and Bork, P. 2002. Common exon duplication in animals and its role in alternative splicing. Hum. Mol. Genet. 11: 1561-1567.

Licatalosi, D.D. and Darnell, R.B. 2006. Splicing regulation in neurologic disease. Neuron 52: 93-101.

Lin, J.C. and Tarn, W.Y. 2005. Exon selection in $\alpha$-tropomyosin mRNA is regulated by the antagonistic action of RBM4 and PTB. Mol. Cell. Biol. 25: 10111-10121.

Liu, Z., Luyten, I., Bottomley, M.J., Messias, A.C., HoungninouMolango, S., Sprangers, R., Zanier, K., Kramer, A., and Sattler, M. 2001. Structural basis for recognition of the intron branch site RNA by splicing factor 1. Science 294: 10981102.

Maeda, I., Kohara, Y., Yamamoto, M., and Sugimoto, A. 2001. Large-scale analysis of gene function in Caenorhabditis elegans by high-throughput RNAi. Curr. Biol. 11: 171-176.

Matlin, A.J., Clark, F., and Smith, C.W. 2005. Understanding alternative splicing: Towards a cellular code. Nat. Rev. Mol. Cell Biol. 6: 386-398.

Matter, N., Herrlich, P., and Konig, H. 2002. Signal-dependent regulation of splicing via phosphorylation of Sam68. Nature 420: 691-695.

Mello, C.C., Kramer, J.M., Stinchcomb, D., and Ambros, V. 1991. Efficient gene transfer in C.elegans: Extrachromosomal maintenance and integration of transforming sequences. EMBO J. 10: 3959-3970.

Mitani, S. 1995. Genetic regulation of mec-3 gene expression implicated in the specification of the mechanosensory neuron cell types in Caenorhabditis elegans. Dev. Growth Differ. 37: 551-557.

Modrek, B. and Lee, C. 2002. A genomic view of alternative splicing. Nat. Genet. 30: 13-19.

Muh, S.J., Hovhannisyan, R.H., and Carstens, R.P. 2002. A Nonsequence-specific double-stranded RNA structural element regulates splicing of two mutually exclusive exons of fibroblast growth factor receptor 2 (FGFR2). J. Biol. Chem. 277: 50143-50154.

Nabel-Rosen, H., Dorevitch, N., Reuveny, A., and Volk, T. 1999. The balance between two isoforms of the Drosophila RNA-binding protein how controls tendon cell differentiation. Mol. Cell 4: 573-584.

Nabel-Rosen, H., Volohonsky, G., Reuveny, A., Zaidel-Bar, R., and Volk, T. 2002. Two isoforms of the Drosophila RNA binding protein, how, act in opposing directions to regulate tendon cell differentiation. Dev. Cell 2: 183-193.

Newman, E.A., Muh, S.J., Hovhannisyan, R.H., Warzecha, C.C., Jones, R.B., McKeehan, W.L., and Carstens, R.P. 2006. Identification of RNA-binding proteins that regulate FGFR2 splicing through the use of sensitive and specific dual color fluorescence minigene assays. RNA 12: 1129-1141.

Orengo, J.P., Bundman, D., and Cooper, T.A. 2006. A bichromatic fluorescent reporter for cell-based screens of alternative splicing. Nucleic Acids Res. 34: e148.

Paronetto, M.P., Achsel, T., Massiello, A., Chalfant, C.E., and 
Ohno et al.

Sette, C. 2007. The RNA-binding protein Sam68 modulates the alternative splicing of Bcl-x. J. Cell Biol. 176: 929-939.

Pettitt, J. and Kingston, I.B. 1994. Developmentally regulated alternative splicing of a nematode type IV collagen gene. Dev. Biol. 161: 22-29.

Ryder, S.P. and Williamson, J.R. 2004. Specificity of the STAR/ GSG domain protein Qk1: Implications for the regulation of myelination. RNA 10: 1449-1458.

Ryder, S.P., Frater, L.A., Abramovitz, D.L., Goodwin, E.B., and Williamson, J.R. 2004. RNA target specificity of the STAR/ GSG domain post-transcriptional regulatory protein GLD-1. Nat. Struct. Mol. Biol. 11: 20-28.

Saccomanno, L., Loushin, C., Jan, E., Punkay, E., Artzt, K., and Goodwin, E.B. 1999. The STAR protein QKI-6 is a translational repressor. Proc. Natl. Acad. Sci. 96: 12605-12610.

Schmucker, D., Clemens, J.C., Shu, H., Worby, C.A., Xiao, J., Muda, M., Dixon, J.E., and Zipursky, S.L. 2000. Drosophila Dscam is an axon guidance receptor exhibiting extraordinary molecular diversity. Cell 101: 671-684.

Schumacher, B., Hanazawa, M., Lee, M.H., Nayak, S., Volkmann, K., Hofmann, E.R., Hengartner, M., Schedl, T., and Gartner, A. 2005. Translational repression of C. elegans p53 by GLD-1 regulates DNA damage-induced apoptosis. Cell 120: $357-368$.

Sibley, M.H., Johnson, J.J., Mello, C.C., and Kramer, J.M. 1993. Genetic identification, sequence, and alternative splicing of the Caenorhabditis elegans a 2(IV) collagen gene. J. Cell Biol. 123: 255-264.

Smith, C.W. 2005. Alternative splicing-When two's a crowd. Cell 123: 1-3.

Smith, C.W. and Nadal-Ginard, B. 1989. Mutually exclusive splicing of $\alpha$-tropomyosin exons enforced by an unusual lariat branch point location: Implications for constitutive splicing. Cell 56: 749-758.

Smith, C.W., Chu, T.T., and Nadal-Ginard, B. 1993. Scanning and competition between AGs are involved in $3^{\prime}$ splice site selection in mammalian introns. Mol. Cell. Biol. 13: 49394952.

Southby, J., Gooding, C., and Smith, C.W. 1999. Polypyrimidine tract binding protein functions as a repressor to regulate alternative splicing of $\alpha$-actinin mutually exclusive exons. Mol. Cell. Biol. 19: 2699-2711.

Stoss, O., Olbrich, M., Hartmann, A.M., Konig, H., Memmott, J., Andreadis, A., and Stamm, S. 2001. The STAR/GSG family protein rSLM-2 regulates the selection of alternative splice sites. J. Biol. Chem. 276: 8665-8673.

Sugnet, C.W., Srinivasan, K., Clark, T.A., O’Brien, G., Cline, M.S., Wang, H., Williams, A., Kulp, D., Blume, J.E., Haussler, D., et al. 2006. Unusual intron conservation near tissueregulated exons found by splicing microarrays. PLOS Comput. Biol. 2: e4.

Ule, J., Stefani, G., Mele, A., Ruggiu, M., Wang, X., Taneri, B., Gaasterland, T., Blencowe, B.J., and Darnell, R.B. 2006. An RNA map predicting Nova-dependent splicing regulation. Nature 444: 580-586.

Venables, J.P. 2004. Aberrant and alternative splicing in cancer. Cancer Res. 64: 7647-7654.

Vernet, C. and Artzt, K. 1997. STAR, a gene family involved in signal transduction and activation of RNA. Trends Genet. 13: 479-484.

Wagner, E.J., Baraniak, A.P., Sessions, O.M., Mauger, D., Moskowitz, E., and Garcia-Blanco, M.A. 2005. Characterization of the intronic splicing silencers flanking FGFR2 exon IIIb. J. Biol. Chem. 280: 14017-14027.

Wicks, S.R., Yeh, R.T., Gish, W.R., Waterston, R.H., and Plasterk, R.H. 2001. Rapid gene mapping in Caenorhabditis el- egans using a high density polymorphism map. Nat. Genet. 28: $160-164$.

Wu, J.I., Reed, R.B., Grabowski, P.J., and Artzt, K. 2002. Function of quaking in myelination: Regulation of alternative splicing. Proc. Nat1. Acad. Sci. 99: 4233-4238.

Yeo, G.W., Nostrand, E.L., and Liang, T.Y. 2007. Discovery and analysis of evolutionarily conserved intronic splicing regulatory elements. PLoS Genet. 3: e85. doi: 10.1371/journal. pgen.0030085.

Zaffran, S., Astier, M., Gratecos, D., and Semeriva, M. 1997. The held out wings (how) Drosophila gene encodes a putative RNA-binding protein involved in the control of muscular and cardiac activity. Development 124: 2087-2098. 


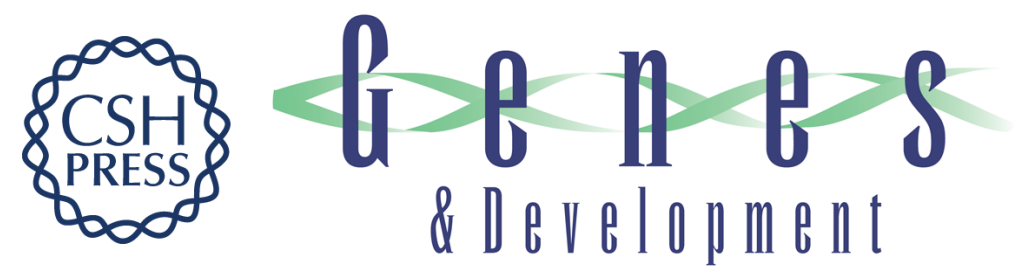

\section{STAR family RNA-binding protein ASD-2 regulates developmental switching of mutually exclusive alternative splicing in vivo}

Genta Ohno, Masatoshi Hagiwara and Hidehito Kuroyanagi

Genes Dev. 2008, 22: originally published online January 29, 2008

Access the most recent version at doi:10.1101/gad.1620608

\section{Supplemental http://genesdev.cshlp.org/content/suppl/2008/01/16/gad.1620608.DC1 \\ Material}

Related Content The search for alternative splicing regulators: new approaches offer a path to a splicing code

Charles J. David and James L. Manley

Genes Dev. February , 2008 22: 279-285

References This article cites 78 articles, 41 of which can be accessed free at: http://genesdev.cshlp.org/content/22/3/360.full.html\#ref-list-1

Articles cited in:

http://genesdev.cshlp.org/content/22/3/360.full.htmI\#related-urls

\section{License}

Email Alerting

Service

Receive free email alerts when new articles cite this article - sign up in the box at the top right corner of the article or click here.

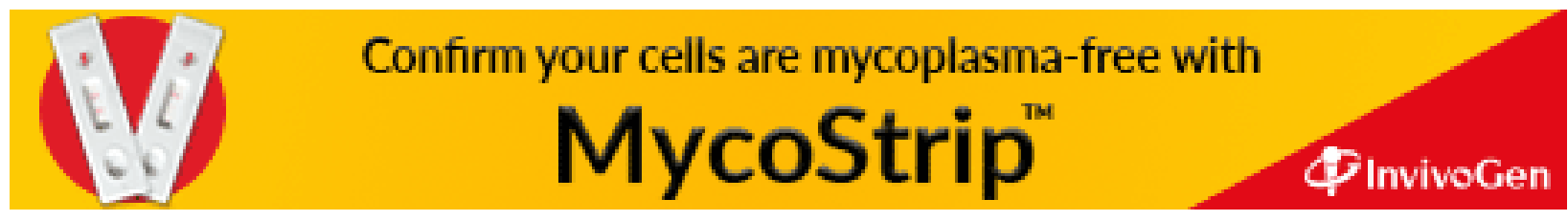

